\title{
UNA ESTRATEGIA DE CAMBIO CONCEPTUAL EN LA ENSEÑANZA DE LA FÍSICA: LA RESOLUCIÓN DE PROBLEMAS COMO ACTIVIDAD DE INVESTIGACIÓN
}

\author{
VARELA NIETO, M.P. ${ }^{1}$ y MARTÍNEZ AZNAR, M.M. ${ }^{2}$ \\ ${ }^{1}$ Instituto Ramiro de Maeztu de Madrid y Departamento de Didáctica de las Ciencias \\ Experimentales de la Universidad Complutense de Madrid. \\ ${ }^{2}$ Departamento de Didáctica de las Ciencias Experimentales de la Universidad \\ Complutense de Madrid.
}

\begin{abstract}
SUMMARY
We present in this paper the main results of a research aimed at the study of a learning/tcaching process directed to familiarise the students with a researching methodology of open problem solving centered in the Physics field. We have studied how this process favours in the students a conceptual change, incardinated in the learning constructivist theory and whether this change lasts with time and is joined by a positive attitude towards the scientific learning.
\end{abstract}

\section{INTRODUCCIÓN}

Desde un punto de vista epistemológico, la ciencia como actividad humana está dirigida fundamentalmente a resolver problemas y, asumiendo que en el campo educativo cualquier disciplina debe ser fiel a su propia naturaleza, en la enseñanza de las ciencias, y en concreto en el caso de la física, la resolución de problemas debe ser contemplada como una tarea básica a realizar por profesores y alumnos. En la línea apuntada, los investigadores en este campo han llevado a cabo un elevado número de trabajos intentando, por un lado, describir modelos sobre cómo los sujetos resuelven problemas, lo que se ha venido en denominar estudios experto-novato (Larkin y Reif, 1979 ; Reif y Heller, 1982; Chi et al., 1988; Eylon y Linn, 1988; López-Rupérez, 1991; Glaser, 1992) y, por otro, desarrollar propuestas metodológicas diseñadas explícitamente para enseñar a los estudiantes a resolver problemas con la característica común de haber cvaluado su nivel de eficacia dentro del aula (Mettes et al., 1980; Caillot y Dumas, 1987; Kramers y Pilot, 1988 y Selveratnam, 1990). Como conclusión de las investigaciones realizadas, podemos decir que existe consenso en el sentido siguiente: Hay que enseñar de forma especifica procedimientos para que los alumnos aprendan a resolver problemas en el campo de las ciencias.
En cuanto a las teorias del aprendizaje que han estado detrás de las prácticas de enseñanza, hasta mediados de los años setenta los trabajos en el caso concreto de las ciencias se apoyaban, en gran medida, en la teoría piagetiana del desarrollo cognitivo. A partir de la segunda mitad de esta década, las aportaciones de Piaget se han visto complementadas por las deducidas de un hecho relevante: la constancia empírica de que los alumnos, antes de acceder a la instrucción formal, han desarrollado un pensamiento propio sobre los fenómenos naturales, comprobándose también que una gran parte de estas ideas permanecen inalteradas. Esta evidencia ha Ilevado a los investigadores y profesores de ciencias a preguntarse sobre el origen de las mismas y su interferencia con el proceso de enseñanza, existiendo en este momento un gran interés por conocer cuáles son esas ideas previas de los alumnos, ya que, según las teorías del aprendizaje, sólo se logrará que éste sea significativo -en términos de Ausubel-si se parte del conocimiento de estas representaciones para construir nuevos esquemas conceptuales.

De acuerdo con to apuntado, la preocupación actual estriba en la posibilidad de que los esquemas de los estudiantes experimenten una determinada evolución 
como consecuencia de procesos educativos y, en este sentido, el problema que se plantea es definir cuáles tienen que ser las características de la instrucción para que esta posibilidad se convierta en realidad. En consecuencia, numerosos equipos de profesores han realizado esfuerzos para diseñar materiales didácticos al efecto (Driver, 1989; Varela et al., 1993) y, concretamente, se han hecho propuestas metodologicas para enseñar a resolver problemas con esta orientación, tal como la que vamos a describir a lo largo de este artículo

\section{LOS ESQUEMAS CONCEPTUALES ALTER- NATIVOS: UN OBSTÁCULO PERTINAZ}

En el marco teórico que estamos comentando, toman relevancia las aportaciones que nos han llegado de dos puntos geográfica y culturalmente muy alejados: la escuela de Moscú representada emblemáticamente por Vygotsky, y la norteamericana a través de la figura de Kelly. Estos autores, junto con otros más próximos a nosotros, entre los cuales es obligatorio mencionar a Piaget y Ausubel, han realizado contribuciones muy interesantes acerca de los procesos de construcción del conocimiento. Sus teorías han colaborado, de forma complementaria, al surgimiento de lo que genéricamente se ha denominado perspectiva constructivista del aprendizaje (Carretero, 1993).

\section{El constructivismo, una teoría emergente}

I.a ciencia, en el marco teórico del constructivismo, aparece como un proceso de interpretación de la realidad mediante la construccion de modelos o a partir de deterrinados paradigmas que la condicionan. Esto significa considerar la ciencia no tanto como un descubrimiento, sino como una contrucción teórica para interpretar el mundo y, en este contexto teórico, aprender ciencia es reconstruir los conocimientos partiendo de las propias ideas de los individuos, ampliándolas o modificándolas según los casos. Otro aspecto, en nuestra opinión muy interesante, es que los teóricos de este modelo consideran los contenidos conceptuales específicos de suma importancia, en contraposición a las corrientes que habían sobrevalorado la importancia de los procesos o destrezas científicas (Millar y Driver, 1987).

Fl constructivismo como teoría emergente ha tenido una fuerte implantación en el campo de la didáctica de las ciencias, en la cual actualmente numerosos autores han desarrollado propuestas de trabajo dentro de este marco ligadas fundamentalmente a procesos de cambio conceptual, procesos que toman como punto de partida los esquemas conceptuales alternativos que poseen los sujetos. El conjunto de investigaciones realizadas presentan notables diferencias no sólo metodológicas, sino también en lo que se refiere al estatus epistemológico que conceden a las ideas alternativas de los alumnos. A pesar del nivel de dispersión apuntado en lo relativo a estas ideas, los autores coinciden en admitir algunas caracte- rísticas comunes que constituirían, en términos de laakatos (1978), el núcleo firme de este enfoque. Estas caracteristicas son las siguientes:

- Su origen se debc a experiencias personales que incluyen la percepción, el lenguaje, el contexto cultural..., teniendo carácter implícito.

- A pesar de ser personales, son comunes en estudiantes de diferentes medios y edades existiendo, en general, unas pocas tipologías en las quc pueden clasificarse la mayor parte de las concepciones alternativas en un área de conocimiento determinada.

- Presentan, en determinados casos, cierto paralelismo con ideas que se han defendido a lo largo de la historia de la ciencia.

- Son bastante estables y resistentes al cambio, por lo que muchas veces persisten a pesar de la instrucción científica.

\section{De las concepciones alternativas al cambio conceptual}

Una vez admitidas y caracterizadas las concepciones alternativas que poseen los alumnos, se plantea cl problema de la evolución de éstas como consecuencia de la enseñanza recibida en las aulas, lo que se ha denominado proceso de cambio conceptual. En este campo, una de las teorías que ha tenido mayor incidencia es la desarrollada por Posner y otros (1982) y que fue ampliada posteriormente por ellos mismos (Hewson, 1992; Hewson y Beeth, 1995). Su fundamentación procede del campo de la filosofía de la ciencia: concibe el aprendizaje cono una actividad racional. Es decir, para estos autores aprender es fundamentalmente llegar a comprender y aceptar las ideas, al ser éstas inteligibles y racionales.

En opinión del grupo de Posner, los puntos de vista contemporáneos de la filosofía de la ciencia sugieren que existen dos fases diferenciadas en el proceso de cambio conceptual en ciencias. El trabajo científico cstá basado en unos compromisos centrales que organizan la investigación; en una primera fase, estos compromisos son los que definen problemas, indican las estrategias a utilizar y especifican los criterios para presentar aquello que se ofrece como solución. Kuhn (1962) denomina a los citados compromisos paradigmas y a la investigación dominada por parađigmas, ciencia normal ; mientas que Lakatos (1978) los califica como su esencia teórica pura, sugiriendo que dichos compromisos generan programas de investigación diseñados para ser aplicados.

La segunda fase del cambio conceptual sucede cuando estos compromisos centrales necesitan alguna modificación. En este caso el científico se enfrenta con un reto a sus supuestos básicos teniendo que adquirir nuevos conceptos y una forma diferente de ver el mundo. Kuhn califica este tipo de cambio conceptual como revolución cientifica y para Lakatos constituye un cambio en los programas de investigación. Fn este sentido, la aparición de una nueva teoría no puede comprenderse en el 
vacío, sino en función de su relación con la teoría anteriormente vigente.

La hipótesis de partida de Posner y otros admite la existencia de pautas análogas a todo lo explicado cuando se considera el cambio conceptual producido en los procesos de aprendizaje. A veces los estudiantes utilizan conceptos que ya poseen para explicar nuevos fenómenos; esta variante de la primera fase del cambio la denominan asimilación. Sin embargo, en otras ocasiones los conceptos preexistentes son inadecuados y los sujetos deben, por tanto, reemplazar o reorganizar sus conceptos centrales; esta forma más radical de cambio conceptual recibe el nombre de acomodación, teniendo en cuenta que al producirse cambios de conceptos centrales no se reemplazan todos a la vez (los autores hacen la salvedad de que, aunque estos términos coinciden con los utilizados por Piaget, su uso no representa un compromiso con sus teorías). Así, las personas retienen muchas de sus concepciones, algunas de los cuales van a funcionar como guía del proceso de acomodación; es lo que se conoce como ecología conceptual del sujeto que proporciona el contexto en que se produce el cambio, influyéndole y confiriéndole significado (Toulmin, 1972).

Todas las ideas presentadas tienen una clara trasposición al contex to escolar, donde el cambio conceptual constituiría una de las metas a conseguir a través del proceso de enseñanza-aprendizaje. El papel del profesor sería propiciar una serie de circunstancias para favorecer la posible evolución (Stofflett, 1994). En nuestra opinión:

1) Hay que conseguir, en primer lugar, que los estudiantes se hagan conscientes de sus propios esquemas.

2) Los alumnos tienen que «enfrentar» sus ideas acerca de un concepto o fenómeno con las aportadas por sus compañeros, el profesor, los resultados de un problema o de un experimento; es decir, provocar en ellos el conflicto cognitivo que proponen Posner y otros.
3) Los estudiantes tienen que tener oportunidades para reestructurar sus conocimientos.

4) Los sujetos han de ser capaces de extender el campo de validez de sus nucvas ideas.

En la actualidad se sigue profundizando en las teorías sobre el cambio conceptual y hay una llamada de atención acerca de la tentación de caer en posturas reduccionistas que no tendrían en cuenta aspectos complementarios del proceso como las formas de razonamiento empleadas por los alumnos, las metodologías utilizadas, los intereses de los sujetos que aprenden, etc. (Furió, 1996; Pozo, 1996).

\section{Los esquemas conceptuales en el campo de la física}

Una vez considerado el marco teórico descrito, y desde una perspectiva práctica, el siguiente paso sería determinar cuáles son los conceptos que resultan claves para abordar un programa básico de física escolar. Tomando como punto de partida los resultados de numerosas investigaciones que ya han sido recopiladas en la literatura sobre el tema, podemos afirmar que cn los campos de la mecánica newtoniana y de la electrocinética se puede conseguir un aprendizaje significativo si los estudiantes consiguen un nivel adecuado acerca de los esquemas que se presentan en la figura 1 y que constituyen los ejes conceptuales de estas materias. Para su explora. ción existen pruebas de lápiz y papel que se pueden considerar validadas, a juicio de expertos (Hierrezuelo y Montero,1989; Gil et a1., 1991; Pfund y Duit, 1994; Varela, 1996).

\section{LA RESOLUCIÓN DE PROBLEMAS: UNA ESTRATEGIA DE CAMBIO CONCEPTUAL}

Las metodologías de trabajo que se pueden utilizar dentro de las aulas, a fin de conseguir un cambio concep-

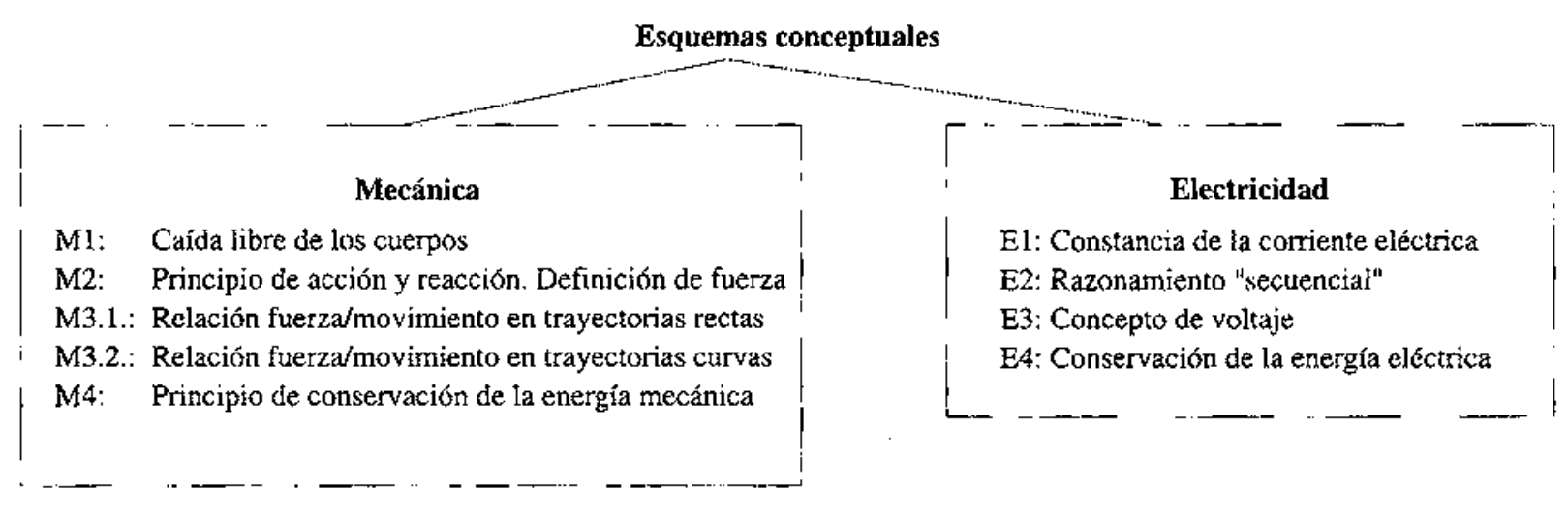


tual en los estudiantes, pueden ser de muy diferente índole y, como ya comentamos en la introducción, la resolución de problemas se presenta como un camino adecuado. En esta línea, una propuesta muy relevante en nuestro país ha sido la elaborada por el equipo de Gil de la Universidad de Valencia, desarrollada con alumnos de bachillerato y profesores en formación inicial y permanente (Gil y Martínez-Torregrosa, 1987; Furió, 1994; Ramírez et al., 1994). El modelo está fundamentado en la comparación entré cómo resuelven los científicos los problemas que se les presentan en el marco de su trabajo y el procedimiento que debe utilizarse dentro de las clases de física y química para que los estudiantes aprendan a resolver sus problemas escolares. En la figura 2 aparece un esquema de las características del modelo.

Figura 2

Características del modelo de resolución de problemas utilizado en esta investigación.

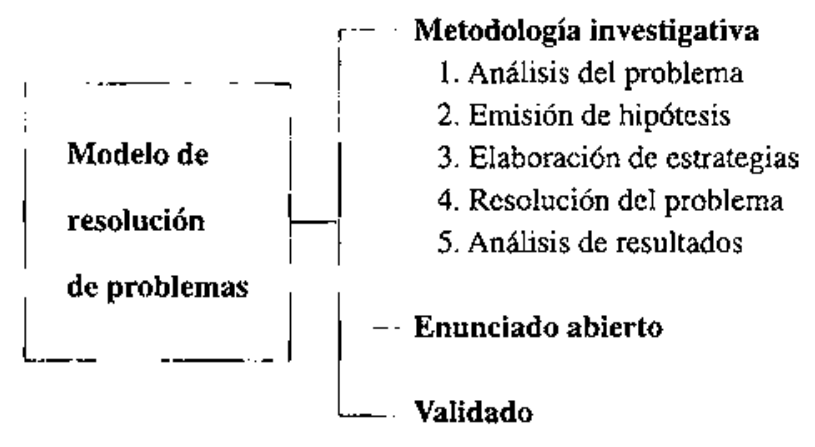

Fn opinón de los autores, que hacemos nuestra, la aplicación a casos concretos de las ctapas de resolución propuestas va a familiarizar a los alumnos con procedimientos propios de la metodología científica. Por otra parte, la utilización de enunciados abiertos y sin datos numéricos plantea situaciones problemáticas que van a permitir a los estudiantes desarrollar procesos de resolución más ricos y complejos que cuando se enfrentan a problemas-ejercicio cerrados, de los usualmente utilizados en nuestras aulas; además, este tipo de tareas fomenta también aspectos relacionados con la creatividad, característica fundamental del trabajo científico (Garret, 1988).

El modelo de resolución de problemas de GiI y otros cumple los requisitos que hemos presentado en el apartado anterior, necesarios para conseguir en los estudiantes un cambio conceptual entendido en el marco de la teoría constructivisa del aprendizaje, ya que hacer un análisis de una situación que se presenta como abierta representa un momento clave para que se hagan conscientes no sólo del problema que se les plantea sino de cuáles son sus concepciones al respecto. La emisión de hipótesis, etapa crucial del proceso, permite a los sujetos explicitar sus ideas acerca de las variables que van a influir, así como de la naturaleza de esta influencia. Por último, el análisis de resultados, la otra "etapa reina" del proceso, favorece la creación de conflictos cognitivos al comprobar la existencia de discrepancias entre lo que se ha hipotetizado y los resultados obtenidos, o bien entre los resultados y el marco teórico en que se ha trabajado, necesitando una revisión crítica del proceso para solucionar el conflicto planteado.

En la línea que estamos comentando, nuestra aportación concreta ha consistido en investigar el cambio conceptual producido como consecuencia del trabajo continuado con el modelo propuesto. De acuerdo con estas premisas, en el diseño y resolución de los problemas se ha insistido en que los alumnos se hagan conscientes de sus ideas sobre un conjunto básico de conceptos de física, planteándoles, a lo largo del proceso de resolución, conflictos que les permitan evolucionar hacia ideas más acordes con la ciencia actual (Varela, 1994).

Para terminar, queremos hacer una llamada de atención sobre el hecho de que las actitudes en el aprendizaje de las ciencias es una cuestión que está adquiriendo gran relevancia al considerarse que los factores cognitivos no son los únicos que mediatizan dicho aprendizaje. En este sentido nosotras hemos partido de la convicción de que un trabajo como el realizado iba a promover en los estudiantes una acritud positiva hacia la resolución de problemas, entenciida ésta como finalidad básica en el aprendizaje citado. En el apartado correspondiente comentaremos los resultados obtenidos.

\section{DISEÑO DE LA INVESTIGACIÓN}

En coherencia con el planteamiento teórico que hemos presentado, nuestro estudio se ha realizado bajo la hipotesis de que la familiarización de los alumnos con el modelo de resolución de problemas descrito va a promover un cambio conceptual, en el sentido de que se va a producir en los estudiantes del grupo experimental, al final del proceso, diferencias significativas con respecto a los esquemas inicialmente disponibles en el campo de la mecánica y de la electricidad.

Por otra parte, hemos estudiado si el nivel de conocimicnto adquirido en los campos explorados va a ser significativamente superior en el grupo experimental -familiarizado con el modelo investigativo de resolución de problemas de enunciado abierto- que en el grupo control, cuyas características describiremos más adelante. Todo esto con la idea de conseguir unos resultados generalizables, fácilmente replicables y, en el campo que estamos trabajando, de claras implicaciones didácticas. En la figura 3 aparece una panorámica general del diseño de la investigación, que permite percibir la com* plejidad del problema planteado.

La muestra objeto de estudio, grupo experimental, ha estado constituida por un grupo intacto de estudiantes de $3^{\circ}$ de BUP ( 36 alumnos de edad media de 16 años y dic $z$ meses), dentro de un contexto escolar normalizado (IB 
Figura 3

Aspectos relevantes sobrc el diseño de esta investigación.

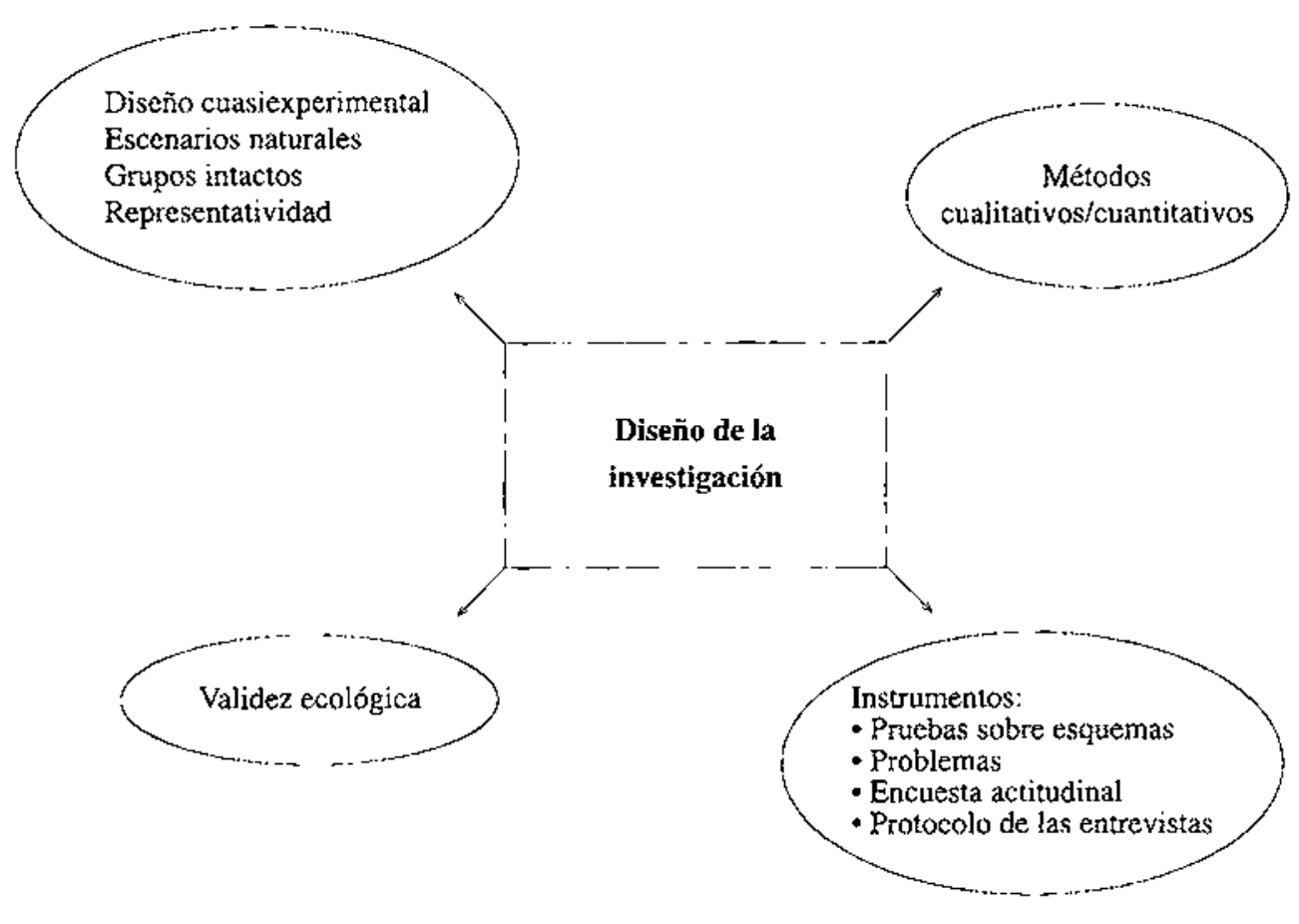

Rey Pastor de Madrid). Como grupo control se escogieron 40 estudiantes, a partir de una muestra formada por 200 individuos, pertenecientes a institutos de bachillerato de Madrid considerados estándar y que iban a realizar un proceso de enseñanza con profesores expertos y metodologías que podemos llamar tradicionales. En toda la elección, el aspecto de la representatividad se ha primado con respecto al problema del tamaño, entendido en el sentido de considerar las muestras seleccionadas como representativas del universo al cual se quieren extrapolar las conclusiones obtenidas de la investigación.

En cuanto a la valide $z$ del diseño nos hemos decantado por una validez ecológica, que se interesa fundamentalmente por la generalización de los efectos experimentales a otras situaciones, permitiendo sacar una serie de conclusiones de tipo educativo, tal como se recogen at final del artículo.

Otra cuestión a destacar es la relativa a las implicaciones curriculares que se derivan de haber trabajado en una situación estándar de clase. Los esquemas conceptuales investigados, enmarcados en la mecánica y la electrocinética (Fig. 1) cubren el programa correspondiente a la física de los dos cursos que actualmente conviven en nuestro sistema educativo: el curso $3^{\circ}$ de BUP y el $1^{\circ}$ de bachillerato de la LOGSE. Su dominio, a través de la resolución de problemas, garantiza un aprendizaje significativo al mismo tiempo que proporciona una base sólida para cursos posteriores.
En esta investigación se ha expiorado cuáles cran tas ideas de los alumnos sobre estos conceptos en el momento inicial, al terminar «la experimentación» y à cabo de diez meses de su finalización.

\section{La metodología dentro del aula, un compromiso en- tre diferentes orientaciones}

EI trabajo dentro del aula se ha desarrollado con una metodología orientada por el paradigma investigaciónacción, donde el profesor juega el doble rol de profesor $\mathrm{y}$ de investigador, participando en el proceso de estudio y mejora de la práctica educativa. En esta perspectiva, la información recogida a través de los mecanismos adecuados ha permitido, a lo largo del proceso, poner en marcha las correspondientes estrategias de realimentacion fundamentales en csta orientación, donde una de las idcas clave es investigar para la acción (Lewin, 1946; Porlan 1987; Elliot, 1990).

Por otra parte, se ha asumido la teoría constructi vista del aprendizaje en la que el propósito de la enseñanzá de la ciencia no es «obligar» a los estudiantes a que cambien sus concepciones alternativas sino más bien ayưaries, tanto a formar el hábito de cuestionar sus ideas como a desarrollar las estrategias adecuadas para contrastar concepciones de cara a su posible accptación. Tomando como punto de partida estas ideas, el trabajo dentro del aula se ba realizado siguiendo las fases que proponen 
Osborne y Freyberg (1985) adaptadas para nuestra situación concrcta del modo siguiente:

- Fase preliminar. El tratamiento comienza con el planteamiento de un problema de enunciado abierto para que los alumnos lo resuelvan trabajando en pequeños grupos. Como era de esperar las aportaciones se producen dispersas y no se consiguen avances importantes. Esta actividad tiene como finalidad no explícita que los alumnos se hagan conscientes de sus propias limitaciones cuando se encuentran un problema de estas características para cuya resolución no tienen las herramientas adecuadas.

- Fase de enfoque. Una ver creado el ambiente necesario, el profesor puede presentar un plan de actuación ante un problema de enunciado abierto. Este plan se concreta en las pautas de trabajo propuestas en el «modelo investigativo"s seleccionado, las cuales reflejan unas orientaciones de tipo directivo, no normativo, propias de la metodología científica.

- Fase de confrontación y aplicación. En este punto los alumnos ya empiezan a estar en condiciones de resolver el tipo de tarea que se les propone. El profesor irá presentando diferentes problemas que, sometidos a discusión en pequeño grupo, pueden pasarse a debate general siguiendo una seric de pasos que consideramos imprescindibles:

a) Para cada una de las situaciones problemáticas que se van planteando, partir de lo que los alumnos piensan al respecto.

b) Dejar el tiempo necesario para que los estudiantes, conscientes de sus propuestas, perciban si éstas conducen o no a resultados satisfactorios entrando en confrontación, al mismo tiempo, con las propuestas de sus compañeros.

c) En este momento el profesor puede aportar sus puntos de vista, siempre planteados a modo de hipótesis, para proteger la situación de conflicto, fundamental en este tipo de metodología.

d) A partir de la confrontación surgida se puede avanzar hacia la resolución del problema, procurando, en la medida de lo posible, llegar a soluciones generales con alto contenido físico. Kil análisis de estas soluciones es un momento fundamental para cuestionat el resuitado obtenido a la luz. de las hipótesis emitidas y de los conocimientos téricos empieados.

e) Si el análisis no resulta satisfactorio, hay que retomar el problema revisando cada uno de los pasos que se han dado. Para la realización de esta revisión es fundamental que todo el proceso haya sido suficientemente verbalizado $\mathrm{y}$, por lo tanto, alejado de tratamientos puramente operativos dificiles de analizar.

Paralelamente a la secuencia descrita, desarrollada dentro del aula, se han introducido en el proceso estrategias de trabajo encaminadas a favorecer en los sujetos aspec- tos que podríamos encuadrar en la línea «aprender a aprenđer», fundamentalmente a partir de entrevistas individuales, unidas significativamente a habilidades metacognitivas (Flavell, 1976; Novak y Gowin, 1988; Moreno, 1989).

\section{El «problema» de la evaluación de los problemas}

Para constatar si Ios estudiantes se han convertido en «resolventes expertos», es decir, en que medida son capaces de resolver individualmente problemas específicos dentro del modelo propuesto, fue necesario diseñar un proccdimiento de evaluación. El asunto se planteó como un probiema complicado, tanto por la modalidad a elegir como por las variables a escoger para proceder a la corrección de las tareas; nos decantamos por una evaluación formativa, entendida como una reflexión crítica sobre todos los momentos y los factores que han intervenido a lo largo del proceso y donde se pretende, fundamentalmente, una mejora de los resuitados y de la racionalidad de la práctica educativa (Rosales, 1981; Santos, 1993 ). Hemos pretendido que la evaluación scà válida, fiable y átil, con la máxima cconomía para el individuo que aprende y para el profesor que está intentando contrastar sus hipótesis.

Para cuantificar el rendimiento de los alumnos se definieron dos tipos de variables denominadas metodológicas y de contenido. Las variables metodológicas (VM), coinciden con las etapas del modelo de resolución propuesto y son comunes, por tanto, a todos los problemas utilizados. Fn el anexo aparece una ejemplificación del procedimiento seguido presentando uno de los problemas empleados en la investigación donde se explicita cómo se aborda cada una de las etapas. En lo que respecta a las variables de contenido (VC), introducidas por razones prácticas, se ha optado por utilizar la noción de esquema de razonamiento que a modo de estructura unitaria configura la resolución del problema $y$, en consecuencia, son específicas para cada uno de los problemas resueltos (López-Rupérez, 1991).

Además, se ha considerado de interés medir el nivel de verbalización, cuantificado en dos categorías que expresan la presencia o ausencia «por escrito» de los razonamientos empleados por los alumnos, puesto que, en la metodología desarrollada en el aula, se enfatiza la necesidad de que verbalicen las operaciones que van realizando. Se ha medido también el grado de abandono, pues nuestra experiencia profesional nos dice que, en numerosos casos, el alumno enfrentado a un probiema de física abandona la tarea en un tiempo muy corto sin tratar de resolverla.

\section{RESULTADOS DE LA INVESTIGACION}

En este apartado vamos a presentar los resultados obtenidos, separando los relativos a la evolución de los 
estudiantes en su capacidad para resolver problemas, de los que hacen referencia al cambio conceptual conseguido por cllos.

\section{¿Se ha favorecido la capacidad de los alumnos para resolver problemas?}

En consonancia con el modelo escogido, se ha evaluado Ia tarea escolar en tres momentos diferentes: inicial o de diagnóstico, de progreso y final, para cada una de las áreas abordadas: mecánica y electricidad. En síntesis, los resultados obtenidos en los seis problemas empleados han sido:

1) Los estudiantes, a medida que se ha ido avanzando en el proceso, han obtenido mejores resultados (aumentos globales del orden del $47 \%$ ). Cuando han cambiado de árca, los individuos han sido capaces de transferir sin dificultad las estrategias de resolución de problemas de una a otra.

2) En el campo de los contenidos, los alumnos han experimentado una evolución global que va desde puntuaciones del orden del $33 \%$ a valores del orden de $77 \%$, tal como se aprecia en la figura 4. La evolución ha sido muy homogénea, no detectándose, en consecuencia, influencia de los contenidos específicos de cada uno de los problemas.

3) En cuanto a las variables metodológicas, relacionadas directamente con las etapas del modelo de resolución en que los estudiantes han sido entrenados, se han observado aumentos del orden del 50\% (Fig. 5), apreciándose, sin embargo, evoluciones diferenciadas: en las đos áreas investigadas, la emisión de hipótesis y el análisis de resultados son las que, relativamente, han experimentado una evolución menor, indicándonos la dificultad que este tipo de tareas presenta para nuestros escolares.

Los resultados obtenidos relativos a la mayor eficacia para resolver problemas coinciden con los aportados en las investigaciones realizadas por Martínez-Torregrosa (1987) y Ramírez (1990), donde los autores concluyen que los alumnos de los grupos experimentales, entrenados en la metodología investigativa de resolución de problemas, obtenían resultados significativamente superiores y de mayor calidad que los pertenecientes a los grupos control.

4) El nivel de verbalización empleado por los estudiantes cuando resuelven problemas ha experimentado un avance importante: al final del proceso, el $86 \%$ de los estudiantes son capaces de verbalizar los procedimentos empleados con un nivel aceptable frente al dato inicial del $5 \%$. Este hecho ha favorecido enormemente las revisiones críticas y el diagnóstico de errores, además de promover estrategias de metaaprendizaje, permitiéndoles, en consecuencia, reflexionar sobre los procesos que están realizando. El otro parámetro contabilizado, $n \dot{u}$ mero de estudiantes que abandona la tarea apenas comenzada, ha sufrido una disminución muy sensible, poniendo de manifiesto que el entrenamiento realizado suministra a los sujetos un conjunto de herramientas utilizable antes de decidirse a abandonar.

5) Por último, hemos explorado las actitudes de los estudiantes hacia el mođelo con que habían estado trabajando, tomando siempre como referencia las metodologías con que ustualmente se resuelven probiemas dentro del aula. Los resultados obtenidos muestran que se ha producido una actitud favorable hacia la tarea de resolver problemas dentro del aprendizaje de la física. En la encuesta realizada, los individuos escogieron en un $68 \%$ de los casos los extremos positivos del diferencial semántico presentado; en particular, cl $80 \%$ de los alumnos considera que se ha favorecido el aprendizaje de conceptos y de procedimientos. En cuanto a la transferencia de lo aprendido a otros úmbitos, un $77 \%$ de la muestra indica la posibilidad de hacerlo a otras áreas de contenido científico y sólo un 35\% lo considera aplicable a la vida cotidiana.

Como síntesis de todo lo expuesto podemos concluir que los estudiantes han experimentado una evolución positiva en cuanto a su eficacia para resolver problemas de enunciado abierto. Esta evolución se produce independientemente del contenido específico de los problemas y ha ido acompañada de una actitud positiva hacia el aprendizaje de la física en general y hacia la resolución de problemas en particular.

\section{¿Qué ha ocurrido en relación con el cambio concep- tual experimentado por los alumnos?}

El supuesto, que a modo de hipótesis nos habíamos planteado, relativo a la posible consecución de un cambio conceptual en los alumnos que habían trabajado con una metodología investigativa de resolución de problemas de enunciado abierto se ha dividido, a efectos prácticos, en tres subhipótesis. Las dos primeras hacen referencia a la evolución de los esquemas conceptuales a medida que va pasando el tiempo; es decir, a cómo las respuestas de los alumnos van acercándose hacia planteamientos más acordes con los defendidos por la ciencia, y si ésta evolución persiste. La tercera subhipótesis se ha reservado para el estudio de la consistencia interna con que utilizan los conocimientos adquiridos.

El estudio global de los resultados pone de manifiesto que en mecánica se ha conseguido un aumento medio del $44 \%$ en el porcentaje de respuestas correctas. En lo relativo a electricidad, el aumento experimentado ha sido del orden del $49 \%$ al final de proceso. Un análisis pormenorizado de los datos muestra que los esquemas más resistentes al cambio conceptual y, consecuentemente utilizados en su forma alternativa por un número importante de alumnos al final del proceso, han sido:

-M32. Relación fuerza-movimiento en trayectorias curvas (existe necesariamente una fuerza en la dirección de la velocidad).

- E2. Uso del razonamiento secuencial (cuando se cambia un elemento de un circuito, la variación producida 
Figura 4

Evolución global de las variables de contenido a lo largo del proceso.

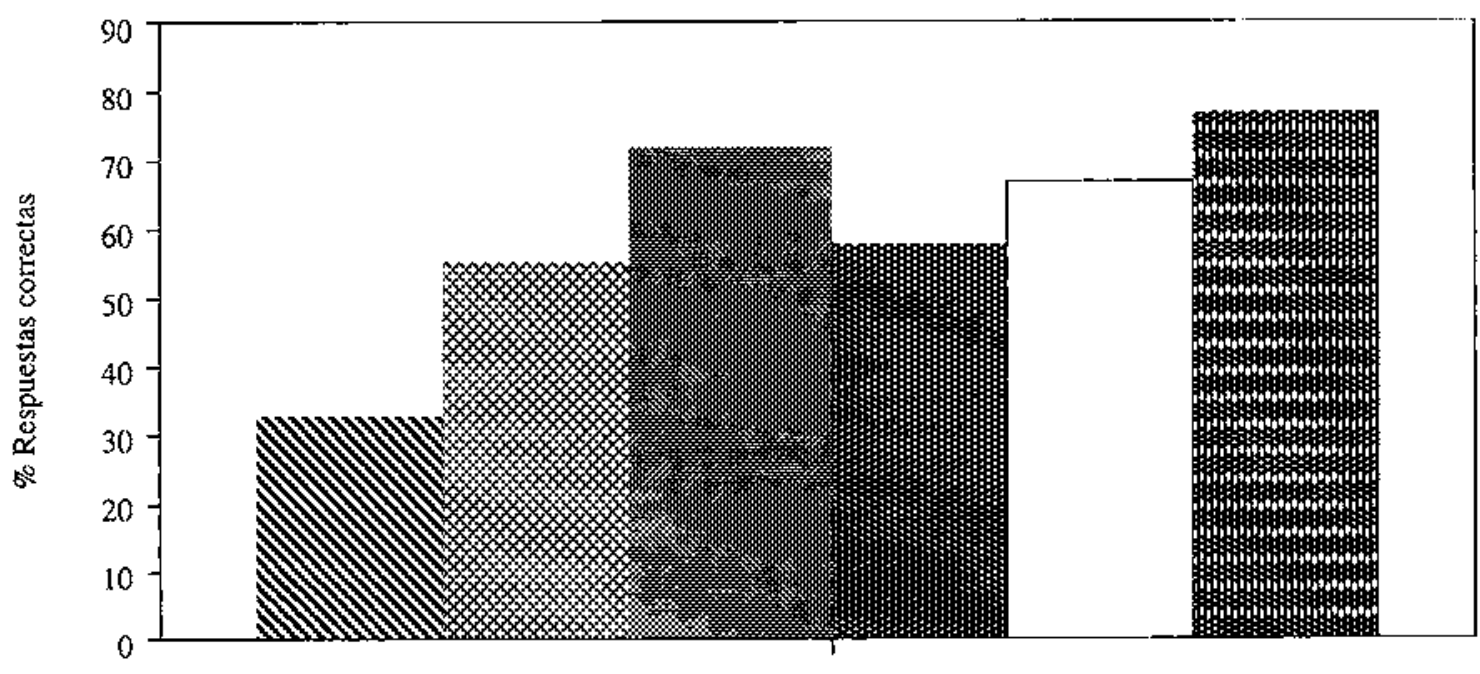

$\begin{array}{lllll}\text { NIIN VC Problema PMI } & \text { VC Problema PM2 } & \text { VC Problema PM3 } \\ \text { 珼聑 } & \text { VC Problema PEI } & \square & \text { VC Problema PE2 } & \text { VC Problema PE3 }\end{array}$

Figura 5

Evolución global de las variables metodoígicas a lo largo del proceso.

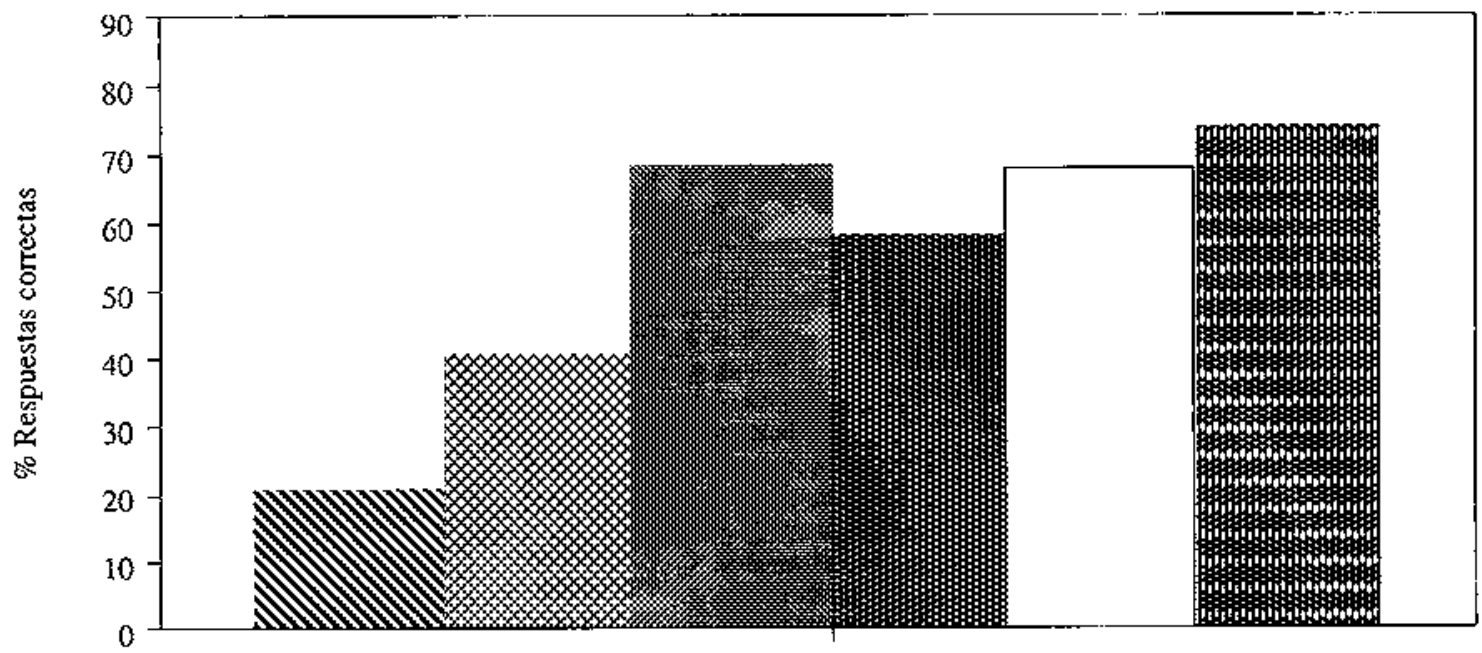

NIt VC Problema PM1

VC Problema PM2

VC Problema PE 1 $\square$ VC Problema PE2

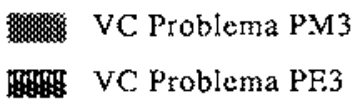


sólo afecta a los elementos que están colocados detrâs del elemento que ha variado).

- E3. Concepto de voltaje (cl voltaje es una consecuencia de que la corriente circule por un circuito y no su causa).

Estos resultados son concordantes con las aportaciones que se han realizado en este campo de investigación y constatan la dificultad de superar la existencia de este tipo de concepciones que, como mencionábamos en el apartado correspondiente, tienen su origen en un análisis que podíamos considerar basado en el sentido común y, por lo tanto, se resisten a ser modificadas (Rhoneck, 1985; Dupin y Joshua, 1986; Sebastià, 1984; Shipstone et al., 1988; Varela et al., 1988, 1996; Gil et al., 1991).

Con el fin de explorar si las diferencias observadas entre la evaluación inicial y final eran significativas desde un punto de vista estadístico, se ha realizado la prueba de Wilcoxon que nos proporciona la dirección de las diferencias, así como la magnitud de las mismas. Los resultados obtenidos para las dos áreas exploradas aparecen en la tabla I y su análisis pone de manifiesto que existen diferencias significativas (nivel de significación $<1 \%$ ) a favor de la situación final para todos los esquemas estudiados, resultado que constituye, sin duda, un éxito importante achacable al modelo utilizado.

En cuanto a la persistencia en el tiempo (uno de los problemas que afectan al cambio conceptual obtenido en

Tabla I

Prueba de Wilcoxon para determinar diferencias inicial/final en los escuemas conceptuales de mecánica y electricidad en el grupo experimental. $p<0,001$.

\begin{tabular}{|l|c|}
\hline Esquemas conceptuales & Wilcoxon \\
\hline Caída libre de los cuerpos. M1 & 4,725 \\
\hline Principio de acción y reacción. M2 & 3,839 \\
\hline $\begin{array}{l}\text { Relación fuerza/movimiento en } \\
\text { trayectorias rectas. M3.1 }\end{array}$ & 4,823 \\
\hline $\begin{array}{l}\text { Relación fuerza/movimiento en } \\
\text { trayectorias curvas. M3.2 }\end{array}$ & 4,326 \\
\hline $\begin{array}{l}\text { Principio de conservación de la energía } \\
\text { mecánica. M4 }\end{array}$ & 4,443 \\
\hline Constancia de la corriente eléctrica. E1 & 5,295 \\
\hline Razonamiento «secuencial». E2 & 4,882 \\
\hline Concepto de voltaje. E3 & 4,939 \\
\hline Conservación de la energía eléctrica. E4 & 5,035 \\
\hline
\end{tabular}

las aulas), los datos tomados al cabo de diez meses de terminado el trabajo con los estudiantes muestran que el cambio conceptual conseguido persiste en el tiempo. Aunque se ha producido un cierto descenso en el porcentaje de respuestas correctas, con un nivel de significa. ción $<1 \%$, no se han producido diferencias significativas para ninguno de los esquemas explorados.

La tercera subhipótesis establecida es la relativa a la evolución de la coherencia interna con que los alumnos utilizan sus esquemas conceptuales. Este aspecto es muy relevante pues una de las finalidades de la enseñanza de la física es conseguir un pensamiento coherente en el marco de esta disciplina. En nuestra investigación se ha determinado la coherencia interna con que se utilizan los esquemas conceptuales mediante el cálculo de los coeficientes de correlación de Kendali, obteniendose al final del proceso los resuitados que aparecen en la tabla II.

El análisis de estos datos muestra que un $81 \%$ de los coeficientes son significativos al $1 \% 0$, con un valor medio de 0,450 ; valores muy superiores a los conseguidos cuando se determinó la coherencia interna en la evaluación inicial. El esquema conceptual correspondiente al principio de conservación de la energía es el que presenta mayor correlación en las dos áreas exploradas. Este resultado pone de manifiesto que el aprendizaje significativo del citado principio es sumanente relevante para la construcción del conocimiento escolar de la física.

Como síntesis podemos decir que los alumnos han logrado al final del proceso un $55 \%$ de respuestas correctas en los esquemas conceptuales investigados, resultado significativamente superior al detectado en la evaluación de diagnóstico, siendo los esquemas que más han evolucionado los referentes a los principios de conservación de la energía mecánica y eléctrica, el estudio de la caida libre de los cuerpos y la constancia de la corriente eléctrica en un circuito. La explicación de esta mejora está, sin duda, en la atención especial que se les ha prestado; así, por ejemplo, el principio de conservación de la energía sc ha aplicado mediante la utilización de problemas a diferentes contextos, incluyendo la idea de degradación, usualmente poco tratada en los currículos escolares. Una asunción correcta de este principio para el caso de los circuitos lleva aparejado el aprendizaje correcto de la constancia de la corriente eléctrica, superando la idea de que algo se va gastando a lo largo del circuito. Para ei caso de la caída de los cuerpos, el hecho de pensar que los cuerpos de mayor masa o peso caen más deprisa que los más ligeros constituye una idea muy intuitiva y, por lo tanto, de díficil erradicación; al igual que en el caso anterior se han realizado diversos problemas para plantear «conflictos» al respecto.

\section{¿Qué hemos encontrado en la comparación con el grupo control?}

Nuestro estudio se había planteado bajo el supuesto de que el cambio conceptual conseguido en el grupo experimental va a ser significativamente superior al conse- 
Tabla II

Coeficientes de correlación de Kendall para el nivel final de los esquemas conceptuales del grupo experimental. $\mathrm{p}<0,001$, excepto en ${ }^{*} \mathrm{p}<0,05$ y ${ }^{* *} \mathrm{p}<0,01$.

\begin{tabular}{|c|c|c|c|c|c|c|c|}
\hline & $\mathrm{M} 1$ & $\mathrm{M} 2$ & $\mathrm{M} 3.1$ & $\mathrm{M} 3.2$ & $\mathrm{E} 1$ & $\mathrm{~F} 2$ & $\mathrm{E} 3$ \\
\hline $\mathrm{M} 2$ & 0,637 & & & & & & \\
\hline $\mathrm{M} 31$ & $0,286^{* *}$ & - & & & & & \\
\hline $\mathrm{M} 32$ & 0,393 & 0,361 & $0,205^{*}$ & & & & \\
\hline $\mathrm{M} 4$ & 0,455 & 0,424 & 0,416 & $-\cdot$ & & & \\
\hline $\mathrm{E} 2$ & & & & & 0,427 & & \\
\hline $\mathrm{l} 33$ & & & & & 0,409 & 0,437 & \\
\hline $\mathrm{F} 4$ & & & & & 0,537 & 0,590 & 0,697 \\
\hline
\end{tabular}

guido con las metodołogías de resolución de problemas habituales en nuestras aulas. Pasadas las pruebas correspondientes a la evaluación de diagnóstico, en lo que a esquemas conceptuales se refiere, y aplicado el estadístico U de Mann-Wihtney para grupos independientes, los resultados mostraron que, en ocho de los nucve esquemas estudiados, no aparecen diferencias significativas, al 1\%o, antes de realizar un tratamiento diferenciado. Este resultado nos permite afirmar que los dos grupos, experimental y control, habían sido tomados de la misma población y es precisamente en clla donde se van a poder extrapolar las conclusiones que de este trabajo se deriven.

Para medir diferencias al final del proceso se ha utilizado de nuevo la prueba U de Mann-Whitney, y los resultados obtenidos aparecen en la tabla III, revelando que existen, en siete de los nueve conceptos estudiados, diferencias significativas, al $1 \%$, a favor del grupo experimental. Un estudio pormenorizado indica que los tres esquemas (M32. Relación fuerza/movimiento en trayectorias curvas, E2. Razonamiento secuencial y E3. Concepto de voltaje), los cuales, como hemos visto en los resultados del grupo experimental, se han mostrado más resistentes ai cambio conceptual, son precisamente aquéllos en los que aparecen las mayores diferencias a favor de este grupo. Este dato corrobora la eficacia de la metodología de resolución de problemas propuesta, en lo relativo a potenciar en los estudiantes el cambio conceptual deseado.

\section{CONCLUSIONES E IMPLICACIONES EDUCATIVAS}

A la vista de los resultados que hemos presentado, podemos sacar como conclusión global que el cambio conceptual conseguido en el grupo experimental en mecánica y en electricidad no solamente es significativo y persistente en el tiempo, sino que es estadísticamente superior al que se obtiene con las metodologías usualmente utilizadas en nuestras aulas.

\section{Tabla IIl}

Prueba $U$ de Mann-Whitney para determinar diferencias en cl nivel final de los esquemas conceptuales entre cl grupo experimental y de control. *p<0.05 ** $\mathrm{p}<0.01 * * \mathrm{*}, \mathrm{p}<0.001$.

\begin{tabular}{|c|c|}
\hline Esquemas conceptuales & $\begin{array}{c}\text { Ude } \\
\text { Monn-Whitney }\end{array}$ \\
\hline Caída libre de los cuerpos. M1 & $2,568^{0}$ \\
\hline Principio de acción y reacción. M2 & $2,393: *$ \\
\hline $\begin{array}{l}\text { Relación fuer } \angle \text { a/movimiento en } \\
\text { trayectorias rectas. M3.1 }\end{array}$ & $2,160^{t a d}$ \\
\hline $\begin{array}{l}\text { Relación fuerza/movimiento en } \\
\text { trayectorias curvas. M3.2 }\end{array}$ & $4,499: 1: \cdots$ \\
\hline $\begin{array}{l}\text { Principio de conservación de la energía } \\
\text { mecánica. M4 }\end{array}$ & $2,793^{3 *}$ \\
\hline Constancia de la corriente eléctrica. El & $1,800^{*}$ \\
\hline Razonamiento «secuencial». E2 & 2,873 ; \\
\hline Concepto de voltaje. E3 & $2,844^{5: *}$ \\
\hline Conservación de la energía eléctrica. E4 & $2,330^{1 * 1 *}$ \\
\hline
\end{tabular}


La explicación del cambio obtenido está, sin duda, en el tipo de metodología de resolución de problemas con que han trabajado los alumnos, en el cual las fases de analisis del problema y emisión de hipótesis les ha permitido explicitar sus ideas previas acerca de los conceptos que iban apareciendo en los problemas, mientras que el análisis de resultados ha favorecido la creación de conflictos cognitivos; ambas circunstancias son fundamentales para la consecución de un cambio conceptual significativo. Además, las estrategias de metacognición utilizadas (verbalización del proceso, contraste con los compañeros y el profesor, entrevistas...) han colaborado sin duda al cambio deseado.

Por otra parte, hay que indicar que el modelo didáctico con el cual hemos trabajado, considerado dentro de un enfoque constructivista del proceso de enseñanza-aprendizaje, ha sido fundamental en la obtención del cambio conceptual, poniendo de manifiesto la pertinencia de asumir la perspectiva constructivista sobre cómo aprenden los sujetos, conceptos científicos y, en consecuencia, aplicarlo a situciones escolares estánđar.

Para terminar, hagamos una reflexión alrededor de la idea de resolución de problemas como eje para desarrollar el currículo escolar de ciencias. Dentro del área de ciencias, la resolución de problemas es reconocida como una actividad escncial, encontrándose integrada en todos los currículos académicos y considerándose, además, instrumento indispensable para la evaluación de los resultados obtenidos por los estudiantes. En este sentido se han venido reatizando en los últimos veinte años un gran número de trabajos sobre el tema y, como consecuencia de ello, está surgiendo una corriente investigadora que intenta fundamentar y potenciar un desarrollo del currículo basado en la resolución de problemas.

Desde la perspectiva del profesor, y recogiendo las ideas del párrafo anterior, un análisis de los resultados obtenidos en esta investigación pone de manifiesto la virtualidad de desarrollar nuestro currículo escolar en lo que a física se refiere, utilizando la resolución de problemas como actividad básica dentro de las clases. El hecho de que los problemas propuestos sean de enunciado abierto, abordándose con una metođología de tipo investigativo, ha familiarizado a los estudiantes con aspectos esenciales del conocimiento científico, desarrollando en ellos estrategias de metaaprendizaje, al mismo tiempo que favorece una actitud positiva hacia la resolución de problemas en el marco general del aprendizaje de las ciencias.

\section{REFERENCIAS BIBIIOGRÁFICAS}

CAILLOT, M. y DUMAS-CARRÉ, A. (1987). PROPHY: Un enseignement de una méthodologie de résolution de problèmes de Physique. Résolution de problèmes en mathématique et en physique. Rapports de recherches, 12, pp.199-244. Paris: INPR.

CARRETERO, M. (1993). Constructivismo y educación. Zaragoza: Edelvives.

CHI, M., GLASER, R. y FARR, M. (1988). The nature of expertise. Nueva Jersey: Hillsdale.

COOK, T.H. y REICHARIJT, CH.S. (1986). Métodos cualitativos y cuantitativos en investigación educativa. Madrid: Morata.

DRIVER, R. (1989). Students' conceptions and the learning of science. International Journal Science Education, 11 , Special Issue, pp. $481-490$.
DUPIN, J.J. y JOSHUA, S. (1986). L'électrocinetique du College à l'Université: evolution des répresentations des élèves, et impact de l'enseignement sur ces répresentations. Bulletin l'Union del Physiciens, 683, pp. 779-800.

EYLON, B.S. y LINN, M.C. (1988). Learning and instruction: An Examination of four rescarch perspectives in Science Education. Review of Educational Research, 58(3), pp. 251-301.

ELLIOT, J. (1990). La investigación-acción en educación. Madrid: Morata.

FLAVELL, J.H. (1976). Metacognitive Aspects of Problem Solving en The Nature of Intelligence. L.B. Resnick (ed.), pp. 231-235. Nueva Jersey: Hillsdale.

FURIÓ, C., ITURBE, J. y REYES, J.V. (1994). Contribución de la resolución de problemas como investigación al paradigma 
constructivista de aprendizaje de las ciencias. Investigación en la Escuela, 24, pp. 89-99.

FURIÓ, C. (1996). Las concepciones altemativas del alumnado en ciencias: dos décadas de investigacion. Alambique, 7, pp. 7-17.

GARRFT, R.M. (1988). Resolución de problemas y creatividad: Implicaciones para el currículo de ciencias. Enseñanza de las Ciencias, 6(3), pp. 224-230.

GHL, D. Y MARTÍNEZ-TORRFGROSA, I. (1987). La resolución de problemas de física. Madrid: Vicens Vives - MEC.

GIL, D., CARRASCOSA, J., FURIÓ, C. y MARTÍNEZ, TORREGROSA, J. (1991). I a enseñanza de las ciencias en la educación secundaria. Barcelona: Honsori.

GLASER, R. (1992).Expert knowledge and processes of thinking, en D.F. Halpern (ed.). Enhancing thinking skills in the sciences and mathematics. Nueva Jersey: Hillsdale.

HEWSON, P.W. (1992). El cambio conceptual en la enseñanza de las ciencias y la formación de profesores. Encuentro «Investigación y Desarrollo del Currículo en la Enseñanza de las Ciencias». Madrid: CIDE.

HEWSON, P.W.y BEETH, M.E. (1995). Enseñanza para un cambio conceptual; ejemplos de fuerza y movimiento. Enseñanza de las Ciencias, 13(1), pp. 25-35.

HIERREZUEL,O, J. y MONTERO, A (1989). La ciencia de los alumnos. Su utilización en la didáctica de la física y la quimica. Barcelona: Iaia.

KRAMERS-PALS, H. y PILOT, A. (1988). Solving quantitative problems guidelines for teaching derived from research. International Journal in Science Education, 10(5), pp. 511 521 .

KUHN, T.S. (1962). The structure of scientific revolutions. University Press, Chicago. Trad. cast. (1975). La estructura de las revoluciones científica. México: Fondo de Cultura Económica.

LAKATOS, I. (1978). The methodology of scientific research programmes: philosophical papers. University Press, Cambridge. Trad. cast. (1983). La metodología de los programas de investigación científica. Madrid: Alianza.

I.ARKIN, J.H., y REIF, F. (1979). Understanding and Teaching Problem-solving in Physics. European Journal Science Education, 1(2), pp. 191-203.

LEWIN, K. (1946). Action-research as minority problems. Journal of Social Issues, 2, pp. 34-36.

LóPEZ RUPÉREZ, F. (1991). Organización del conocimiento $y$ resolución de problemas en física. Madrid: Centro de Publicaciones del MEC.

METTES, W., PILOT, A. ROOSSINK, H.J. y KRAMERSPALLS, H. (1980). Teaching and Learning Problem solving in Science. Part I. A general strategy. Journal of Chemical Education, 57(12), pp. 882-885.

MARTÍNEZ,-TORREGROSA, I. (1987). La resolución de problemas de física como investigacion: Un instrumento de cambio metodológico. Tesis doctoral. Universidad de Valencia.

MILLAR, R. y DRIVER, R. (1987). Beyond processes. Studies in Science Education, 14, pp. 33-62.
MORENO, A. (1989). Metaconocimiento y aprendizaje escolar. Cuadernos de Pedagogia, 173, pp. 53-58.

NOVAK, J. y GOWIN, D. (1988). Aprendiendo a aprender. Barcelona: Martínez-Roca.

OSBORNE, J.R. y FREYBER,G.P. (1985). Leaming in Science. The implications of children's science. Londres: Heinemann. Trad. cast. (1991). El aprendizaje d $e^{\prime}$ las ciencias. Madrid: Narcea

PFUND, H. y DUJIT, R. (1994). Students' Alternative Frameworks and Science Education. Kiel, Alemania: Institute for Science Education.

POSNER, G., STRIKE, D., HEWSON, P. y GLR'TZOG, W. (1982). Accomodation of a scientific conception: Toward a theory of conceptual changc. Science Education, $66(2)$, pp. 211-227.

PORLÁN, R. (1987). El maestro como investigador cn el aula. Investigar para conocer, conocer para enseñar. Investigar en la Escuela, 1, pp. 63-70.

POZO. J.I. (1996). Las ideas del alumnado sobre la ciencia: de donde vienen, a dónde van... y mientras tanto qué hacemos con ellas. Alambique, 7, pp. 18-26.

RAMÍREZ, L. (1990). La resolución de problemas de física y química como investigación en la enseñanzá media: un instrumento de cambio metodológico. Tesis doctoral. Universidad Autónoma de Barcelona.

RAMÍREZ, L., GIL, D. y MARTÍNEZ- TORREGROSA, I. (1994). La resolución de problemas de física y quimica como investigación. Madrid: Centro de Publicaciones MEC.

REIF, F. y HELLER, I.I. (1982). Knowledge structure and problem solving in physics. Educational Psychologist, 17, (2), pp.102-127.

RHONECK, C. (1985). The introduction of voltage as an independent variable. The importance of preconceptions, cognitive conflict and operating rules. Aspects of understanding Electrcity. Proceedings of an International Workshop, Ludwisburg IPN Kiel, pp. 275-286.

ROSALES, C. (1981). Criterios para una evaluación formativa. Madrid: Narcea.

SANTOS, M.A. (1993).La evaluación: un proceso de diálogo, comprensión y mejora. Investigación en la Escuela, 20, pp. 23-35.

SEBASTIA, J.M. (1984). Fuerza y movimiento: Ia interpretación de los estudiantes. Enseñanza de las Ciencias, $2(3)$, pp.161169 .

SELVERATNAM, M. (1990). Problem-solving, a model approach. Education in Chemistry, 27(6), pp. 163-165.

SHIPSTONE,D.M., RHONECK, C., KARRQVIST,C., DUPIN, J.y LICHT, P. (1988). A study of students understanding of elcctricity in five European countries. International Joumal Science Education, 10(3), pp. 303-316.

STOFFLETT, R.T. (1994). The accommodation of Science Pedagogical Knowledge: The Application of Conceptual Change Constructs to Teacher Education. Joumal of Research in Science Teaching, 31(8), pp.787-810. 
TOULMIN, S. (1972). La comprensión humana I. El uso colectivo y la evolución de los conceptos. Madrid: Alianza.

VARELA, M.P. (1994). La resolución de problemas en la enseñanza de las ciencias. Aspectos didácticos y cognitivos. Tesis doctoral. Universidad Complutense de Madrid.

VARELA, M.P. (1996). Las ideas de los alumnos en física. Alambique, 7, pp. 45-52.
VARELA, M.P., MANRIQUE, M. J. y FAVIERLS, A. (1988). Circuitos cléctricos: una aplicación de un modclode enseñanzaaprendizaje basado en las ideas previas de los alumnos. Enseñanza de las Ciencias, 6(3), pp. 285-290.

VARELA, M.P., FAVIERES,A., MANRIQUE, M. J. y P. DF LANDAZÁBAL, M. C. (1993). Iniciación a la física en el marco de la teoría constructivista. Madrid: Centro de Publicaciones del MEC.

[Artículo recibido en febrero de 1996 y aceptado en octubre de 1996.] 


\section{JUGANDO FN UNA RAMPA}

Se lanza un objeto por una pendiente. ¿Hasta qué punto conseguirá ascender por el lado opuesto?

Fste problema admite dos estrategias de resolución diferentes. Una, aplicando la segunda ley de Newton para calcular las aceleraciones en cada tramo y a partir de ahí, y con las ecuaciones del movimiento, calcular velocidades y espacios. Ia otra, aplicando el principio de conservación de la energía mecánica que aunque se podría realizar tomando únicamente los puntos inicial y final, los alumnos objeto de este estudio, se han hecho siempre por tramos. Vamos a presentar a continuación la solución de] problema.

\section{Variables metodológicas}

VM1. El análisis cualitativo consiste en describir que el objeto se va a mover sobre una trayectoria como la dibujada:

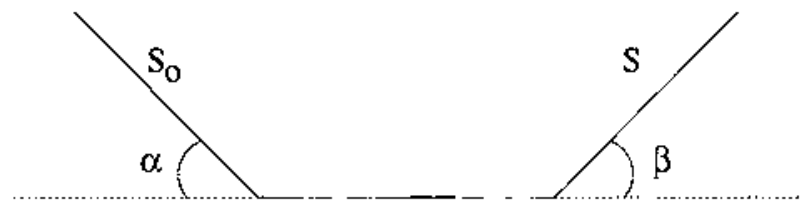

Lin el análisis cualitativo, algunos estudiantes concretan la probiemática presentada en el caso: una bola bajando por un carril, un cochecito tirado por una rampa, movimientos semejantes a los del juego del minigolf, etc.

A partir de considerar un objeto con una velocidad inicial en el punto más alto de una de las rampas, hay que reconocer que subirá por el lado opuesto hasta que se pare; es decir, hasta que su velocidad sea cero.

La restricción que mayoritariamente van a introducir nuestros alumnos es que podamos despreciar el rozamiento. Hay que tener en cuenta que en este nivel académico el manejo de las fuerzas de rozamiento en planos inclinados presenta para ellos una dificultad nada despreciable. También se puede admitir la restricción de que las dos rampas presenten la misma inclinación.

VM2. La emisión de hipótesis tiene aquí varios aspectos que vamos a describir.

Primer tramo: La velocidad con que llega al punto más bajo va a depender directamente de la velocidad con que salió y de la inclinación de la rampa, aparte de la gravedad. Es muy frecuente que los alumnos introduzcan como variable influyente la masa del objeto.

Segundo tramo: Si no hay rozamiento, la velocidad va a permanecer constante independientemente de la longitud del camino horizontal.

Tercer tramo: El espacio recorrido va a depender directamente de la velocidad inicial e inversamente de la inclinación de la rampa. Aquí suele aparecer también la masa retrasando la subida: a mayor masa, antes se parará.

Un caso límite de fácil comprensión es que, si la inclinación de la rampa de subida vale cero, el cuerpo continuaria moviéndose a velocidad constante a lo largo del plano horizontal.

VM3. Como ya hemos comentado antes, este problema admite dos estrategias de resolución, habiendo realizado los estudiantes ambas en la resolución de este problema.

VM4. La resolución del problema en el caso de aplicar las leyes de Newton para el primer tramo, nos lievará a obtener para la velocidad adquirida el valor de:

$$
v=\sqrt{v_{0}^{2}+2 a s}
$$


donde:

$$
a=g \cdot \operatorname{sen} d \alpha
$$

en el $2^{\circ}$ tramo, si despreciamos el rozamiento, la velocidad permanecerá constante y, para el tercero, el espacio recorrido hasta pararse será:

$$
s=\frac{v^{2}}{2 a}
$$

donde ta aceleracion vale $a=g \cdot \operatorname{sen} \beta$. Sustituyendo su valor y el de la velocidad con que inició la subida, quedará para el espacio recorrido antes de pararse:

$$
s=s_{0} \frac{\operatorname{sen} \alpha}{\operatorname{sen} \beta}+\frac{v_{0}^{2}}{2 g \operatorname{sen} \beta}
$$

Si el problema se resuelve por energías, la velocidad al final del primer tramo sería:

$$
v=\sqrt{v_{0}^{2}+2 g_{0}}
$$

esta velociđad se mantendría a lo largo del tramo horizontal y la aitura final alcanzada hasta pararse resultaría:

$$
\mathrm{h}=\mathrm{h}_{0}+\frac{\mathrm{v}_{0}^{2}}{2 \mathrm{~g}}
$$

VM5. El análisis de resultados para este problema presenta varias facetas. Resuelto el problema por los dos procedimientos, es muy interesante comprobar que se liega al mismo resultado, ya que se cumple:

$$
\begin{aligned}
& h_{0}=s_{0} \operatorname{sen} \alpha \\
& h=s \cdot \operatorname{sen} \beta
\end{aligned}
$$

y sustituyendo en la expresión de las alturas queda:

$$
s \cdot \operatorname{sen} \beta=s_{0} \operatorname{sen} \alpha+\frac{v_{0}^{2}}{2 g}
$$

de donde despejando $s$ obtenemos:

$$
\mathrm{s}=\mathrm{s}_{0} \frac{\operatorname{sen} \alpha}{\operatorname{sen} \beta}+\frac{\mathrm{v}_{0}^{2}}{2 \mathrm{gsen} \beta}
$$

que coincide con el resultado obtenido mediante el otro procedimiento utilizado.

Por otra parte, a la luz de las hipótesis avanzadas se puede comprobar que $s$ depende directamente de la velocidad inicial con que se lanza el cuerpo así como de las características de la rampa $\left(s_{0}\right.$ y $\alpha$ o su combinación $\left.h_{0}\right)$. Efectivamente el ángulo $\beta, 0$ más exactamente $\operatorname{sen} \beta$, influye inversamente en el valor alcanzado para el espacio s. Si no se considera el rozamiento, la longitud del tramo horizontal no influye en el resultado. 


\section{Variables de contenido}

Si se procede a una resolución dinámica tenemos:

VC1. Dibujar ejes de coordenadas en los tramos que constituyen la trayectoria. Dibujar las fuerzas que actían en cała tramo, realizando, para los planos inclinados, la descomposicjón gráfica de las mismas.

VC2. Resolver el primer tramo aplicando la segunda ley de Newton y reconociendo la existencia de velocidad inicial y una fuerza resultante hacia abajo.

VC3. Reconocer que en el tramo horizontal la únjca fuerza que afecta al movimiento es el rozamiento, en cuyo caso la velocidad disminuiría. Si se ha considerado que no existe rozamiento, el movimiento será uniforme y la velocidad permanecerá constante a lo largo del plano.

VC4. Aplicar de nucvo la segunda ley de Newton que, en este caso, debido a la existencia de una fuerza resultante cr senticio contrario a la velocidad, conduce a un movimiento desacelerado hasta pararse. El espacio recorrido o la altura alcanzada será el resultado del problema.

Para el caso de resolver el problema por conservación de la energía, tenemos las siguientes variables de contenido:

VC1. Aplicar el principio de conservación de la energía mecánica del primer tramo reconociendo que inicialmente háy encrgía cinćtica y energía potencial que van a convertirse íntegramente (si no hay rozamiento) en energía cinética cn cl punto de abajo.

VC2. En el tramo horizontal, si no se considera el rozamiento, la energía mecánica se conserva y, por lo tanto, llega al final del tramo con la misma velocidad.

VC3. Asumir que el punto más alto al que llegará el móvil conesponderá a una energía potencial igual, por conservación, a la encrgía cinética con que inició la subida. Deducir la altura alcanzada. 